\title{
Pseudoexfoliation of the lens in Australian Aborigines
}

\author{
H. R. TAYLOR, \\ From the Department of Ophthalmology, University of Melbourne, Melbourne, Victoria \\ F. C. HOLLOWS, AND D. MORAN \\ From the Department of Ophthalmology, University of New South Wales, Sydney, New South Wales
}

SUMMARY The first cases of pseudoexfoliation of the lens to be found in Australian Aborigines are reported. The prevalence rate in the communities in central Australia is $1.3 \%$ and for those 61 years and older is $16.3 \%$. Within the limits of our assessment glaucoma was an associated finding in $8.1 \%$ of these cases. A high incidence of old trachoma, previous trauma, and Labrador keratopathy was also noted in these people.

Pseudoexfoliation of the lens (PXF) is a degenerative ocular condition, characterised by the deposition of a fluffy white material on the anterior lens capsule and on the surfaces of the anterior chamber, especially the pupil margin. It is often associated with the dispersion of pigment granules on the corneal endothelium and the trabecular meshwork, and with chronic open-angle glaucoma.

The incidence of PXF has been found to vary greatly in different races and different geographical areas (Duke-Elder, 1969; Aasved, 1969). This paper reports the first documented cases of PXF among Australian Aborigines, and presents the prevalence rate for this condition as it occurs in central Australia. The patients were identified during examinations undertaken by the National Trachoma and Eye Health Programme, which is assessing the ocular status of Aboriginal communities.

\section{Materials and methods}

A routine ocular examination under field conditions was performed on most members of Aboriginal communities in northern South Australia (see map). Visual acuity was assessed, and anterior segment examination for trachoma grading was made, using a $\times 3$ loupe. If ocular disease was identified, the patient was examined on the slit lamp and ophthalmoscopy was done. Intraocular tension was measured by Goldmann applanation tonometry

Address for reprints: Dr H. R. Taylor, Department of Ophthalmology, 32 Gisborne Street, East Melbourne, Victoria, 3002, Australia where possible. In other cases, where applanation was impracticable because of field conditions, tensions were measured with the Schiotz tonometer or digitally. Perimetry was not performed.

\section{Results}

A total of 2773 Aborigines were examined. This represents $80 \%$ of the estimated Aboriginal population of these communities. Alice Springs was

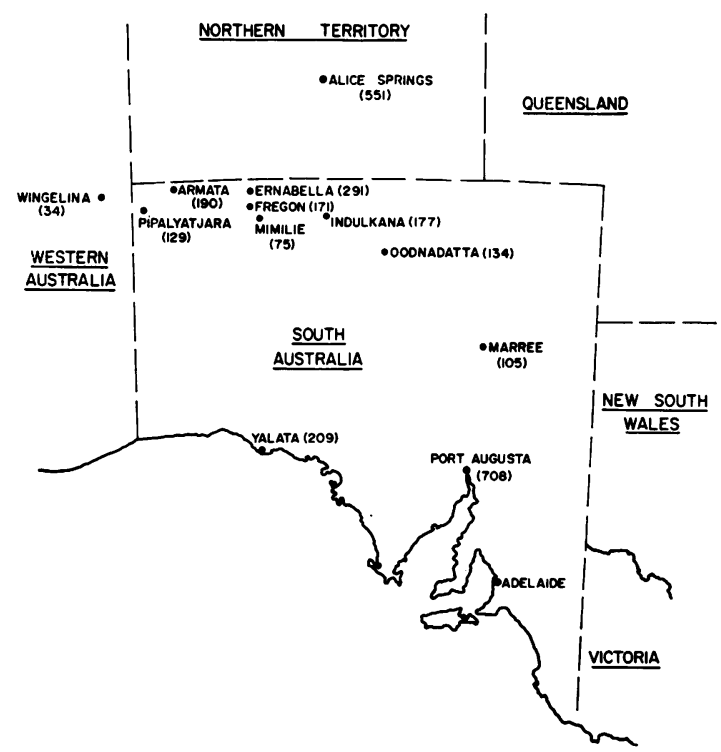

Aboriginal communities studied. The number of people in each community is shown in brackets 
excluded; only an initial survey has been undertaken there comprising $40 \%$ of the Aboriginal population. All cases were from members of the Pitjantatjara or related tribes; and all, except 2 cases, were thought to be full-blood Aborigines.

Clinical details of 37 cases of PXF are shown in Table 1. The distribution of PXF according to age is shown in Table 2. Pseudoexfoliation was not found in any person under 30 years old.

Every patient showed stigmata of trachoma, usually manifested as a superior corneal pannus. Nine people had Labrador keratopathy; 6 phthisical eyes were found; and in 1 case an eye had been enucleated. Three patients had had a unilateral cataract extraction; in 1 of these spontaneous resorption of the lens had occurred in the fellow eye. One case showed lens-induced uveitis. The rest of the patients, with the exception of 7 cases, had moderate to dense nuclear or cortical lens opacities in each eye.

Three cases (Nos. 24, 30, and 32) showed evidence of glaucoma; 1 case (No. 25) had elevated IOP, but the discs were not visible; and 2 cases (Nos. 19 and 28) had abnormally cupped discs and normal tensions.

\section{Discussion}

This is the first report of PXF in the distinct racial group of Australian Aborigines. Of the 2773 people examined 184 were over 60 years old. This gives a prevalence rate of $16.3 \%$ in this age group of 61 years upwards. The prevalence rates for younger

Table 1 Summary of clinical findings in 37 cases of pseudoexfoliation found in Australian Aborigines

\begin{tabular}{lcc}
\hline Clinical finding & $\begin{array}{l}\text { Number } \\
\text { of cases }\end{array}$ & Percentage \\
\hline Raised IOP* with normal disc & 1 & $2 \cdot 7$ \\
Raised IOP with abnormal disc & 3 & $8 \cdot 1$ \\
Normal IOP with abnormal disc & 2 & $5 \cdot 0$ \\
Lens opacities & 30 & $81 \cdot 0$ \\
Dislocated lens & 6 & 16.0 \\
Labrador keratopathy & 9 & $24 \cdot 0$ \\
\hline
\end{tabular}

*IOP = intraocular pressure.

Table 2 Distribution of PXF in Aborigines according to age

\begin{tabular}{lccc}
\hline Age in years & Total examined & Total with PXF & $\begin{array}{l}\text { Percentage } \\
\text { with PXF }\end{array}$ \\
\hline $0-30$ & 2044 & 0 & 0 \\
$31-40$ & 246 & 1 & $0 \cdot 4$ \\
$41-50$ & 193 & 3 & $1 \cdot 6$ \\
$51-60$ & 106 & 3 & $2 \cdot 8$ \\
$61+$ & 184 & 30 & $16 \cdot 3$ \\
Total & 2773 & 37 & $1 \cdot 3$ \\
\hline
\end{tabular}

age groups are $0.4 \%$ for 31 to 40 years, $1.6 \%$ for 41 to 50 years, and $2.8 \%$ for 51 to 60 years, no PXF being found in patients of less than 30 years.

These figures are interesting when they are compared with other prevalence figures in the literature. Luntz (1972) reported cases of PXF from the glaucoma unit in Johannesburg, South Africa, which showed an extrapolated prevalence rate of $0.001 \%$ in the White community, and $0.006 \%$ for the Bantu community. This was interesting, for, as he points out, other authors (Grable and Sugar, 1947) have considered PXF to be rare in American Negroes, who share a common gene pool with the Bantu. Our figures are most striking as we find an overall prevalence in the community of $1.3 \%$, which is 300 times the prevalence found in the Bantu. Moreover, our figures are based on the examination of $80 \%$ of most communities and are not extrapolated from a glaucoma clinic population.

The prevalence in the over-60 age group is $16 \cdot 3 \%$. This is more than three times that found by Aasved (1969) in an examination of 2058 cases of the same age group from the cities of Bergen, Birmingham, and Bonn. His overall prevalence of PXF was $5.0 \%$.

Glaucoma was found in association with PXF infrequently. Although we were limited in our ability to perform a full examination for glaucoma (in particular the lack of visual field testing), elevated intraocular pressure and pathological disc cupping was found in 3 cases. One case of unilateral ocular hypertension was found. This occurred in the affected eye of a patient with unilateral PXF. Two patients, with large optic discs (disc ratios in No. 19 of $0.4,0.4$; and in No. 28 of 0.3 , not visible) and relatively low intraocular pressures (in No. 19 of $17,17 \mathrm{mmHg}$; and in No. 28 of $20,20 \mathrm{mmHg}$ ) were seen. In 1 (No. 19) the PXF and abnormal cupping were bilateral; the other case (No. 28) had unilateral PXF with abnormal cupping in the affected eye, the other disc could not be seen because of lens opacities. At present the distribution of ocular pressure in Australian Aborigines is not known. When this distribution is known, the pressures recorded in these cases may prove to be significantly raised. Thus the prevalence rate of raised intraocular pressure in PXF-affected eyes was $10 \cdot 1 \%$ and of glaucoma of $8 \cdot 1 \%$. Luntz (1972) reported raised IOP in $79 \%$ of Bantu with PXF and $50 \%$ of Whites. In a study of the Norwegian population Aasved (1971) assessed the risk of eyes with PXF developing glaucoma as 20 to $25 \%$.

In each case of raised pressure, the drainage angles were open on gonioscopy. As yet, no cases of chronic simple open-angle glaucoma have been reported in Australian Aborigines. 
Pseudoexfoliation was found to occur predominantly in older patients; however, the youngest patient was only 35 years old. The youngest reported patient was 31 (Horven and Hutchinson, 1967). As the exact age of many of the older patients was not known, no attempt has been made to calculate the average age of the group and compare it with that of other series. Only 10 of the 37 cases occurred in females. Duke-Elder (1969) and Aasved (1969) state that there is no appreciable sex preference, although others have found a preponderance of males (Luntz, 1972; Gradle and Sugar, 1947).

In 21 cases PXF occurred bilaterally. In 7 of the 16 unilateral cases the unaffected eye was either phthisical, showed gross corneal scarring, or had been enucleated. One of the remaining unilateral cases had well-formed Kruckenberg spindles in both eyes. Seven of these unilateral cases involved the right eye and only 2 involved the left. Luntz (1972) also reported a preponderance of unilateral cases involving the right eye. The significance of this is not known. It is of interest that cases 6 and 7 were brothers. Aasved (1969) has summarised the occasional familial occurrence of PXF. Pseudoexfoliation was commonly associated with cataracts. Thirty patients had moderate to dense cataracts. In 6 of these there was also subluxation or dislocation of the lens.

Every patient showed stigmata of trachoma to a greater or less degree, and in this regard they reflected the general findings in the communities. The findings related to the prevalence and severity of trachoma in Aboriginal communities will be the subject of later reports. A high incidence of posttraumatic sequelae is noted, and reflects that seen in others of the same age group. Labrador keratopathy was also a frequent finding, and is seen commonly in older Aborigines. It is thought to be the end result of chronic exposure to bright sunlight and an arid environment (McGuiness et al., 1972).

It will be interesting to see if the similar extremely high incidence of PXF will be found in other Aboriginal groups as the survey progresses, or whether it reflects a condition confined to the Pitjantatjara. If it is an isolated phenomenon, it would add great weight to the theory of a genetic basis for PXF.

The authors wish to thank members of the National Trachoma and Eye Health Programme for their assistance, in particular, Dr W. E. Gillies; and to express their appreciation for the co-operation shown by members of the Aboriginal communities visited.

The National Trachoma and Eye Health Programme is sponsored by the Royal Australian College of Ophthalmologists and funded by the Commonwealth Department of Health, under the provision of the National Health Insurance Act.

\section{References}

Aasved, H. (1969). The geographical distribution of fibrillopathia epitheliocapsularis. Acta Ophthalmologica, 47, 792-810.

Aasved, H. (1971). The frequency of fibrillopathia epitheliocapsularis in patients with open angle glaucoma. Acta Ophthalmologica, 49, 194-210.

Duke-Elder, S. (1969). System of Ophthalmology, Vol. XI. Diseases of the lens and vitreous: glaucoma and hypotony, p. 46. Kimpton: London.

Gradle, H. S., and Sugar, S. (1947). Glaucoma capsulare. American Journal of Ophthalmology, 30, 12-19.

Horven, I., and Hutchinson, B. T. (1967). Exfoliation syndrome. Acta Ophthalmologica, 45, 294-298.

Luntz, M. H. (1972). The prevalence of pseudoexfoliation syndrome in an urban South African clinic population. American Journal of Ophthalmology, 74, 581-587.

McGuiness, R., Hollows, F. C., Tibbs, J., and Campbell, D. (1972). Labrador keratopathy in Australia. Medical Journal of Australia, 2, 1249-1250. 